\title{
EDGE-CONNECTIVITY AND EDGES OF EVEN FACTORS OF GRAPHS
}

\author{
NAStaran Haghparast and Dariush Kiani \\ Department of Mathematics and Computer Sciences \\ Amirkabir University of Technology, Tehran, Iran \\ e-mail: nhaghparast@aut.ac.ir \\ dkiani@aut.ac.ir
}

\begin{abstract}
An even factor of a graph is a spanning subgraph in which each vertex has a positive even degree. Jackson and Yoshimoto showed that if $G$ is a 3-edge-connected graph with $|G| \geq 5$ and $v$ is a vertex with degree 3 , then $G$ has an even factor $F$ containing two given edges incident with $v$ in which each component has order at least 5 . We prove that this theorem is satisfied for each pair of adjacent edges. Also, we show that each 3-edge-connected graph has an even factor $F$ containing two given edges $e$ and $f$ such that every component containing neither $e$ nor $f$ has order at least 5. But we construct infinitely many 3 -edge-connected graphs that do not have an even factor $F$ containing two arbitrary prescribed edges in which each component has order at least 5 .
\end{abstract}

Keywords: 3-edge-connected graph, 2-edge-connected graph, even factor, component.

2010 Mathematics Subject Classification: 05C70, 05C45.

\section{REFERENCES}

[1] J.A. Bondy and U.S.R. Murty, Graph Theory with Applications (North-Holland, NewYork-Amsterdam-Oxford, 1982).

[2] N. Haghparast and D. Kiani, Even factor of bridgeless graphs containing two specified edges (2017), Czechoslovak Math. J., submitted.

[3] B. Jackson and K. Yoshimoto, Even subgraphs of bridgeless graphs and 2-factors of line graphs, Discrete Math. 307 (2007) 2775-2785.

doi:10.1016/j.disc.2006.11.023 
[4] B. Jackson and K. Yoshimoto, Spanning even subgraphs of 3-edge-connected graphs, J. Graph Theory 62 (2009) 37-47.

doi:10.1002/jgt.20386

[5] F. Jaeger, A note on sub-Eulerian graphs, J. Graph Theory 3 (1979) 91-93. doi:10.1002/jgt.3190030110

[6] M. Kano, C. Lee and K. Suzuki, Path and cycle factors of cubic bipartite graphs, Discuss. Math. Graph Theory 28 (2008) 551-556.

doi:10.7151/dmgt.1426

[7] H.-J. Lai, Eulerian subgraphs containing given edges, Discrete Math. 230 (2001) 63-69.

doi:10.1016/S0012-365X(00)00070-4

Received 28 April 2017

Revised 28 August 2017

Accepted 28 August 2017 